\section{$\underset{\substack{\text { hommes } \\ \text { \& migrations }}}{ }$}

\section{Hommes \& migrations}

Revue française de référence sur les dynamiques

migratoires

1286-1287 | 2010

Les migrations subsahariennes

\title{
Des voix sur la solidarité internationale
}

Voyage au cœur d'une association, Entretien avec les salariés du GRDR

\section{Patrick Gonin}

\section{(2) OpenEdition}

1 Journals

\section{Édition électronique}

URL : http://journals.openedition.org/hommesmigrations/1718

DOI : 10.4000/hommesmigrations. 1718

ISSN : 2262-3353

Éditeur

Musée national de l'histoire de l'immigration

Édition imprimée

Date de publication : 1 juillet 2010

Pagination : 16-23

ISSN : 1142-852X

\section{Référence électronique}

Patrick Gonin, « Des voix sur la solidarité internationale », Hommes \& migrations [En ligne],

1286-1287 | 2010, mis en ligne le 29 mai 2013, consulté le 03 mai 2019. URL : http://

journals.openedition.org/hommesmigrations/1718; DOI : 10.4000/hommesmigrations.1718

Tous droits réservés 


\section{Des voix sur la solidarité internationale \\ Voyage au cour d'une association Entretien avec les salariés du GRDR}

Par Patrick Gonin, géographe, université de Poitiers, Migrinter, administrateur du GRDR

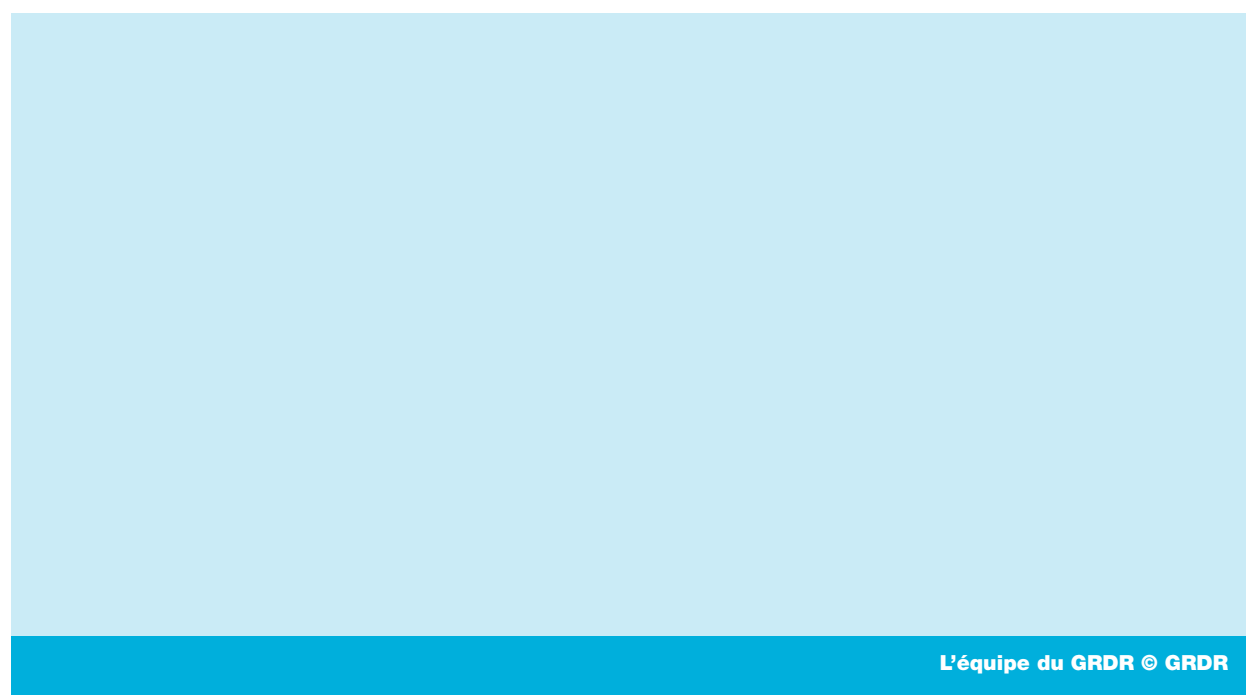

Le GRDR est une organisation professionnelle de solidarité internationale, engagée depuis quarante ans en France et dans quatre pays ouest-africains (Mali, Mauritanie, Sénégal et tout dernièrement Guinée Bissau) autour des thématiques suivantes : migrations, citoyenneté et développement. Pour présenter son histoire mais aussi son public, 14 salariés qui exercent diverses activités ont été rencontrés. Un débat riche traverse les salariés du GRDR, il a été saisi sur le vif et n'engage pas l'association. Cette dernière travaille

à l'élaboration d'une charte qui se voudra une synthèse des valeurs et des orientations politiques partagées entre toutes les parties prenantes

(adhérents, salariés, association de migrants, ONG locales, partenaires stratégiques). Cette charte guidera l'action du GRDR pour les dix prochaines années. 
Le GRDR affiche, pour ses quarante ans, une nouvelle devise : Les migrations, une ressource durable pour des territoires solidaires." Mais les mots des salariés du GRDR pour caractériser leur "employeur" sont multiples: "Une ONG professionnelle, technique avec une faible dimension politique", "une école du développement qui transfere des compétences". Deux images sont également proposées, celle de la tortue "par sa longévité et son histoire, par son rythme lent dans le changement, par sa carapace et sa vulnérabilité", et celle de la ruche, car "elle bourdonne didées, de travail collectif et sait faire du miel et le partager". Nous retiendrons également la construction suivante : "le GRDR est une association professionnelle du développement, promotrice de citoyenneté, de solidarité et de liens de proximité". Les activités décrites par les acteurs de cette table ronde concernent des domaines d'intervention (santé, éducation, hydro-agricole...), des actions de capitalisation, d'accompagnement, d'éducation, mais aussi d'articulation entre le développement local et la sécurité alimentaire ou la lutte contre les discriminations et les actions socio-sanitaires. En fait, l'action du GRDR se traduit "par la création d'outils de planification et de maîtrise du contexte local [...], par l'accompagnement technique et méthodologique des acteurs [du développement]".

\section{Un public cible qui change}

Les migrations subsahariennes à la fin des années cinquante avaient pour origine les régions rurales du bassin du fleuve Sénégal et étaient exclusivement masculines. Elles sont depuis devenues aussi urbaines et féminines. Selon certains salaries, "beaucoup de partenaires n'ont pas toujours pris en compte les nouvelles données: les migrations de la côte (Afrique centrale, Togo, Côte d'Ivoire) ou les deuxièmes générations. Les problématiques ne sont pas les mêmes. C'est donc avec un peu de surprise et de résistance qu'on a vu le glissement des anciennes problématiques aux nouvelles : on est passé de l'intégration/lutte contre le racisme à la lutte contre les discriminations/pour la diversité. La gestion d'inégalités transversales dans un contexte d'absence d'immigration, d'une mixité dans la pauvreté, doit nous amener à de nouvelles postures, parce que dans les prochaines années, notre public aura muté." Ainsi, "pour investir ces nouvelles mobilisations qui ne sont plus communautaires, il [faudra] que le GRDR reste en veille et poursuive son travail d'expérimentation au niveau des jeunes et des nouveaux migrants."

Il semble nécessaire de reconnaître que "les associations, partenaires historiques $d u$ GRDR et très axées sur les projets villageois, existent toujours mais n'ont plus le dynamisme d'antan. Cette assertion est pourtant à nuancer car les associations portent actuellement des projets nettement plus importants qu'auparavant. Ce ne sont plus des puits ou des dispensaires, mais des postes de santé, des collèges, des systèmes d'adduction d'eau complets, 
des lycées, des centres de formation, des radios communautaires, etc. Ces projets nécessitent plus de moyens financiers et techniques. Des leaders nettement plus outillés techniquement prennent le dessus dans les bureaux des associations. Pour autant, ces leaders vieillissent et le renouvellement des générations n'est pas assuré. En effet, nous observons l'arrivée de nouvelles associations (pas si nombreuses) de jeunes nés en France ou arrivés récemment (regroupement familial ou primo-arrivants). Ces associations souhaitent porter des projets comme leurs aînés, mais elles se mobilisent finalement plus sur des activités de sensibilisation auprès de leurs concitoyens français ou d'expressions culturelles. Il ne s'agit pas là d'une relève pleine et entière car ces associations restent rares dans le paysage."

Le GRDR ne risque-t-il pas de s'enfermer dans un rôle de "gestionnaire" des projets et des populations liées aux migrations du bassin du fleuve Sénégal ? Il faut reconnaitre sa capacité à innover malgré le fait que "lévolution des pratiques $d u G R D R$ a été fortement influencée par le positionnement des partenaires financiers et leurs thématiques du moment, les relations migrants-villageois, les enjeux de l'immigration au Nord et du développement dans les zones d'intervention du GRDR [au Sud]. [Ce salarié] constate quatre périodes dans cette évolution des pratiques : la première phase où l'immigré est au centre des activités du GRDR (formation projets collectifs, dynamiques associatives, retours volontaires) - le GRDR était la principale voire l'unique association travaillant sur la thématique migration-développement. La deuxième phase [date] de 1981 avec le développement des initiatives des migrants, la naissance de grosses fédérations, des investissements forts des migrants, le foisonnement des associations, l'accompagnement de leaders associatifs et le développement avec les activités du GRDR au Sud. Au début des années quatre-vingt-dix, le débat au sein du GRDR est lancé sur les relations migrants-villageois, sur la nécessité aussi de tenir davantage compte des initiatives des villageois; le GRDR Afrique commence à s'étoffer. Dans les années deux mille, il investit le champ du développement local et les questions de citoyenneté. Le GRDR s'est professionnalisé, il n'est plus seul sur le champ de la migration et du développement, il tend à devenir une ONG classique du Nord avec une grande plus-value : sa forte présence au Sud à travers les cellules."

\section{Une évolution organique}

Sa longue histoire reste encore à interroger: "Le GRDR a 40 ans, autant de programmes, une centaine de salariés, une dizaine de sites dintervention [...]. Les pratiques $d u$ GRDR ont évolué en fonction des contextes et des demandes des partenaires [...]. Cette capacité d'adaptation du GRDR peut être considérée comme l'un de ses atouts majeurs. Elle est l'une des rares structures dans le bassin du fleuve Sénégal qui s'adapte à toutes les situations et sur des thématiques diverses." Cette évolution est certainement une prise de 
risque obligée. Cette ONG a débuté par "un accompagnement très technique des migrants [pour proposer] une vision de plus en plus politique de la migration. Et, plus récemment, l'accompagnement en France des associations de migrants au quotidien est peu à peu remplacé par un accompagnement de fédérations, de réseaux, etc."

"Le GRDR est dans un processus d'adaptation organique, car il est fortement déterminé, dans son évolution, à la fois par ses transformations internes induites par les femmes et les hommes qui le font (adhérents, administrateurs, salariés, bénévoles) et par son interaction avec les migrants. Organique, également, car le GRDR conçoit sa pratique entre professionnalisme et engagement militant aux côtés de ses partenaires que sont les migrants et leurs territoires de vie et d'origine." Pour un autre salarié, "le GRDR a [su] élever son niveau d'analyse des contextes et de son cadre d'intervention. Il était temps de sortir de l'échelle villageoise et de l'addition de projets ponctuels. Le GRDR serait mort à l'heure actuelle si cette évolution n'avait pas eu lieu. Le cadrage des actions avec la décentralisation ou en lien direct avec des enjeux nationaux, comme la gestion du foncier ou la sécurité (souveraineté) alimentaire, [est une bonne chose]. L'augmentation des marges de mancuvre des antennes et cellules et la professionnalisation des équipes, tout en maintenant une logique assez militante, expliquent la bonne évolution du GRDR. À [ses] yeux, en France, les activités n'ont pas su changer l'échelle d'intervention, se connecter avec les enjeux européens et capitaliser sur les pratiques. [Et] le problème de la capitalisation est central au GRDR. Beaucoup trop d'expériences et de savoir-faire reposent uniquement sur les histoires personnelles des salariés. Au final, la structure est fragile [...]. Avec le recul de l'histoire [il est] heureux de ses évolutions, car le GRDR du passé (années quatre-vingt et quatre-vingtdix) ne [lui] semble pas être une structure si intéressante."

\section{Développement et solidarité : de nouveaux enjeux}

Quarante années de pratiques, d'engagements et de réalisations pour/avec/aux côtés des populations subsahariennes de l'Afrique de l'Ouest. Ce temps reste court face aux enjeux qu'il s'est fixés, mais dans certains cas la "tortue" se déplace trop lentement : "Le GRDR demeure une ONG technicienne et techniciste (la technique peut régler les problèmes). L'ONG tente néanmoins, depuis une période récente (années deux mille), de se servir des références produites à travers la mise en cuvre de différents projets pour construire un discours et porter un plaidoyer solide et crédible sur la sécurité alimentaire (domaine d'activité historique du GRDR) et les migrations (à travers le prisme du double espace). Malheureusement, il [lui] semble que le GRDR (comme la diaspora du bassin du fleuve Sénégal) reste très marqué par l'idéologie du développement telle qu'elle est véhiculée depuis 
la Seconde Guerre mondiale par les Nations unies, le FMI la banque mondiale... [et de suggérer à ses adhérents, administrateurs, salariés, bénévoles de lire l'ouvrage de] G. Rist : Le Développement, histoire d'une croyance occidentale."

Le GRDR a-t-il vocation à "s'éterniser", à ne travailler qu'avec des migrants ou des populations discriminées? Ce qui semble certain, c'est cette capacité de l'organisation à faire rêver ou, à l'opposé, douter sur son avenir: "Le GRDR revendique un ancrage local fort, inégalé dans cette partie de l'Afrique. Par la promotion de ce lien quasi organique avec les associations de migrants - qui se sont elles-mêmes organisées à toutes les échelles [...], le GRDR peut donner un autre sens à la solidarité internationale et construire un projet de société oxygéné autour de l'idée de ce double-espace multiterritorial à la richesse encore insuffisamment explorée..." Mais un autre salarié suggère une autre réflexion tout aussi impertinente: "Le GRDR a accompagné les migrants dans les projets pour là-bas, il s'est développé en Afrique pour appuyer des initiatives locales. Ici, il accompagne le vieillissement. Comme il n'ira pas fleurir les tombes, il faut quil redéfinisse son public ici en tenant compte de sa mutation, sans oublier son identité. Les politiques se préparent aux violences urbaines nées d'un sentiment d'abandon. Elles n'associent plus uniquement la problématique à l'immigration, mais aux précarités."

\section{La recherche d'une place entre collectifs et individus}

Les migrants subsahariens de l'ouest de l'Afrique sont connus pour leurs implications dans des actions collectives, mais le projet migratoire est d'abord et avant tout une affaire individuelle et familiale. Quelles lectures ont les salariés des possibles articulations entre projets individuels et projets collectifs? Un salarié remarque qu' il "serait opportun de s'interroger [sur] la place de l'accompagnateur : devant, derrière, à côté ? Dit autrement il s'agit de s'interroger [sur le rôle de ces] accompagnateurs techniques [...]. Le GRDR enferme des acteurs dans des logiques de projets. Dans quelle mesure participe-t-il, au même titre que d'autres acteurs (la famille, le village, les élus communaux, les coopérations décentralisées du Nord, les bailleurs internationaux, les politiques de décentralisation...), à une prise au piège [...] où l'intérêt individuel serait mis au service d'un intérêt collectif?'

Cette articulation entre les projets collectifs et les initiatives individuelles fait débat et des solutions sont envisageables : "Il y a possibilité de faire en sorte que des projets individuels soient promus à partir de projets collectifs, même si beaucoup de projets ne sont pas conçus comme ça. Il y avait, par exemple, un fonds de crédit dans les années quatrevingt-dix qui a permis à des individus des zones ORDIK et Diama Djigui dans le cercle de 
Kayes de lancer des projets économiques individuels. Les résultats ont conduit à l'élaboration du projet PASECA (microfinance). Le PAIDEL 3 [un autre programme du GRDR] dans son axe 3 pourrait stimuler les initiatives entrepreneuriales." Ce salarié pense qu'il serait utile de "développer les articulations entre projets individuels et collectifs." Mais cette recherche d'articulation est confrontée à une autre difficulté : "Il sera de plus en plus difficile d'associer les deuxièmes générations dans les projets menés là-bas. Ils n'ont pas le même parcours et encore moins le même projet. Certains se sont complètement coupés de leur pays d'origine. Notre approche devra tenir compte de nouveaux enjeux."

\section{Promouvoir la valeur ajoutée des migrants}

Des programmes soutenant les initiatives individuelles ont existé par le passé, mais leur public ne concernait pas uniquement les Subsahariens et les zones d'intervention du GRDR. "Comment éviter de refaire les erreurs du passé et laisser instrumentaliser les migrants en actionnant le double levier de la culpabilité (redevabilité et devoir moral vis-à-vis du pays d'origine) ou de la fierté d'agir qui flatte l'ego. L'autre écueil est également de considérer le migrant dans sa dimension uniquement économique et de travestir la réalité de l'environnement économique ou la résumer au juste apport financier. Dès lors, le GRDR, qui a réamorcé le travail d'évaluation de la demande tant individuelle que collective des migrants à l'aune des enjeux des territoires d'origine comme d'accueil, se doit de parfaire son expertise tant sur le rôle d'interface entre l'offre et la demande qu'en aiguisant son rôle de plaidoyer et de sensibilisation des parties prenantes."

Cette recherche d'articulation entre les initiatives collectives et privées semble à l'heure actuelle quasiment impossible "dans la mesure où les structures associatives ne pensent pas ce lien et ne souhaitent pas le penser. Ces associations [partenaires du GRDR] freinent largement les initiatives individuelles." Mais, là encore, d'autres salariés sont plus optimistes. Il est alors expliqué que "le parcours migratoire est, pour un migrant du bassin du fleuve Sénégal, la résultante d'une infinité de connexions entre projets individuels et collectifs, au cceur desquelles la famille joue un rôle fondamental. C'est à travers l'analyse - pas toujours simple! - des différentes destinations de l'épargne individuelle que s'appréhendent le mieux ces liens : mandats mensuels pour l'achat des produits de premières nécessités, envois spécifiques pour une fête ou une maladie, investissement dans la construction d'un logement, alimentation des caisses de solidarité pour les mutuelles de santé ou les rapatriements de corps, ou enfin les cotisations spécifiques pour des projets associatifs (construction de mosquées, d'écoles, de postes de santé, de banques de céréales...), il s'agit toujours de l'épargne individuelle des travailleurs migrants en Europe (estimée jusqu'à 33 \% des revenus mensuels) ou en Afrique. 
Conscient de cela, le GRDR travaille à valoriser les plus-values de la migration dans les processus de développement des territoires d'origine comme des territoires d'accueil. L'objectif est aussi de diminuer tant que possible les moins-values qui leur sont liées (fuite de la maind'euvre, assistanat, changement d'habitudes alimentaires, inflation, oisiveté...). La porte d'entrée pour l'association étant de ne pas aborder la mobilité sous l'angle des 'remises' mais bien des 'valeurs ajoutées' de la mobilité, perçue comme une ressource durable pour des territoires solidaires." Il s'avère que les projets collectifs ne sont jamais la somme des projets individuels. Cependant, afin de réaliser les intentions collectives pour l'intérêt de ceux qui ne sont pas partis, les porteurs de ce type de projet ont besoin des investissements individuels, de manière à assurer la pérennité du projet.

\section{Le GRDR au cœur d'un double espace}

Les notions pour signifier les liens de dépendance entre émigration et immigration se sont multipliées ces dernières années (transnationalisme, circulation migratoire...). Le GRDR porte la notion de double espace, mais sa compréhension par ses salariés est encore en devenir.

Pour rendre compte de ce débat, ont été retenus les propos de quatre salariés. La première citation en fournit une définition possible; la deuxième rappelle cette histoire tout en pointant d'autres champs dont celui de la citoyenneté ; la troisième citation pointe également des éléments de son histoire et tente de traduire cette construction par deux exemples ; la dernière propose de déconstruire cette notion. "Le double espace est une représentation conceptuelle, imagée, de l'espace migratoire, comprenant plusieurs territoires (d'origine, de transit et de vie) et qui reflète les modes de vie des migrants du bassin du fleuve Sénégal autour de l'articulation migration-développement: bien vivre ici pour mieux être là-bas, garder le lien avec là-bas pour être bien ici! Il y a autant de double-espace que de migrants, mais il est possible de lui donner davantage corps dans des dynamiques de coopération décentralisée, de territoire à territoire, d'acteurs à acteurs..." "L'action du GRDR et de ses partenaires migrants sont à l'origine de nombreuses interactions entre des communes $d u$ Nord et des villages du Sud (ensuite des communes) à travers des jumelages coopération. Ce type de coopération, qui va évoluer au fil des années, a des incidences sur le positionnement des migrants ici et sur la perception des villageois sur leur place et sur le rôle des migrants. Les dynamiques organisationnelles ont renforcé les liens ici et là-bas. Rappelons que lors de la rencontre de Dakar pour le trentième anniversaire du $G R D R$, ce sont les représentants des associations villageoises qui ont posé la question de la deuxième génération et insisté pour que le GRDR intervienne sur les questions d'insertion. Le double espace au GRDR, pour moi, c'est l'interface entre ici et là-bas assurée par une 
ONG. Pour le migrant, notamment celui de la première génération, je mettrai l'accent sur cette double appartenance ou citoyenneté qui se traduit par son investissement sur le plan politique, social et économique dans sa commune d'origine et celle d'accueil."

\section{Promouvoir des interactions entre territoires}

"Lorsque l'on travaille au GRDR, ces interactions sont évidentes. Que serait le bassin du fleuve Sénégal sans l'appui de la migration? Je ne parle pas des infrastructures sociales de base, mais de toute l'histoire de la mobilisation sociale de ces zones. Dans le même temps, en France, ce rôle de 'responsable' du territoire d'origine, avec en toile de fond un fonctionnement communautaire très poussé, a nettement handicapé l'intégration sociale et économique des migrants du bassin du fleuve Sénégal. Ces interactions peuvent bien entendu être très positives sur les deux territoires simultanément, comme lorsque les associations partenaires des territoires d'origine s'impliquent plus sur leur territoire de vie en France." "Cette notion rejoint celle d'interaction spatiale. En termes d'exemples, je peux citer l'association Nouvel Espoir de Thialy qui, autour d'un projet au pays, a permis à des jeunes français d'affirmer leur identité 'multiple', de se rapprocher de leurs parents, de rencontrer des partenaires, de se sentir utiles, de créer une vraie dynamique associative rassemblant tous les jeunes de ce village en France et donc de mieux se connaître aussi, etc. J'ai participé aussi à la naissance de la coopération décentralisée entre [une commune malienne] et [une intercommunalité de France]. Les premiers contacts entre les migrants et la population de ce territoire rural étaient très impressionnants. D'un côté, c'était la première fois que des gens parlaient à des migrants, comprenaient ce qu'impliquait un itinéraire migratoire et, de l'autre, des migrants présents en France depuis vingt ans, sortaient pour la première fois de Paris et engageaient des relations d'amitié qui durent encore. Côté Mali, ce partenariat (pas très efficace en réalité) a permis de renforcer l'adhésion des populations derrière la commune récente." "Pour comprendre ce qui se passe dans les parcours migratoires et dans les sociétés d'accueil et de départ, il faut penser conjointement à la fois émigration et immigration, mais il s'agit là d'une posture de pensée et non d'un concept. D'où la nécessité d'avoir une approche 'pluridimensionnelle' (et pas seulement uni ou bidimensionnelle) qui prenne en compte l'ensemble de ces interactions. Le 'double espace' est un concept avant d'être une réalité de terrain. Plus encore, à mon sens, il s'agit d'un concept qui n'est pas opératoire et qui ne permet pas de rendre compte des interactions spatiales et sociales dans lesquels sinscrivent les migrants ou les jeunes d'ascendance immigrée." Si ces propos sont contradictoires, ces "dissensus" doivent être perçus comme une preuve de vitalité pour cette ONG. 